Original Research Paper

\title{
Isolation, Identification and Genomic Analysis of Plesiomonas shigelloides Isolated from Diseased Percocypris pingi (Tchang, 1930)
}

\author{
${ }^{1,2,3}$ Lei Pan, ${ }^{4}$ Shuiyi Liu, ${ }^{1}$ Xuwei Cheng, ${ }^{1}$ Yiting Tao, ${ }^{5}$ Tao Yang, \\ ${ }^{5}$ Peipei Li, ${ }^{1,2}$ Zhengxiang Wang, ${ }^{3}$ Dongguo Shao and ${ }^{6}$ Defeng Zhang \\ ${ }^{1}$ School of Resources and Environmental Science, Hubei University, \\ Wuhan 430062, People's Republic of China \\ ${ }^{2}$ Hubei Key Laboratory of Regional Development and Environmental Response (Hubei University), \\ Wuhan 430062, People's Republic of China \\ ${ }^{3}$ State key Laboratory of Water Resources and Hydropower Engineering Science (Wuhan University), \\ Wuhan, 430072, People's Republic of China \\ ${ }^{4}$ Department of Medical Laboratory, the Central Hospital of Wuhan, Tongji Medical College, \\ Huazhong University of Science and Technology, Wuhan 430014, People's Republic of China \\ ${ }^{5}$ Wuhan Heyuan Green Biological Co., Ltd., Wuhan 430206, People's Republic of China \\ ${ }^{6}$ Key Laboratory of Fishery Drug Development, Ministry of Agriculture, Pearl River Fisheries Research Institute, \\ Chinese Academy of Fishery Sciences, Guangzhou, People's Republic of China
}

\author{
Article history \\ Received: 25-10-2017 \\ Revised: $11-12-2017$ \\ Accepted: 20-12-2017 \\ Corresponding Author: \\ Defeng Zhang \\ Key Laboratory of Fishery Drug \\ Development, Ministry of \\ Agriculture, Pearl River Fisheries \\ Research Institute, Chinese \\ Academy of Fishery Sciences, \\ Guangzhou, People's Republic of \\ China \\ Email: zhangdefeng08@126.com
}

\section{Introduction}

Plesiomonas shigelloides is a Gram-negative, motile, non-spore-forming, oxidase-positive and rod-shaped bacterium belonging to the Enterobacteriaceae family (Brenner et al., 2005). P. shigelloides is widely distributed in the aquatic environment even under cold climatic conditions (Salerno et al., 2010). Thus far, P. shigelloides has been isolated from streams, lakes, estuarine waters, mammals, crustaceans, mollusks, reptiles, amphibians and fish (Alexander et al., 2016). P. shigelloides is an

\begin{abstract}
Recently, the outbreak of a serious infectious disease of unknown etiology was noted in Percocypris pingi (Tchang, 1930) farms in Yunnan province. Due to currently limited information, we aimed to identify the pathogen isolates, determine the susceptibility of the isolates, evaluate the pathogenicity and analyze the genome of the representative strain. Ten strains of Gram-negative rods were isolated from diseased $P$. pingi and the isolates were identified as Plesiomonas shigelloides based on biochemical characteristics, 16S rRNA gene sequencing and speciesspecific PCR detection. The results of susceptibility analysis showed that wo selected strains LS1 and LL2 were resistant to ampicillin, penicillin G, trimethoprim/sulfamethoxazole, tetracycline, enrofloxacin, nalidixic acid and enoxacin. A virulence assay indicated that the pathogen was virulent to zebrafish. Genomic analysis revealed that the LS1 isolate was cosely related to strain GN7, which was isolated from animal farms. To the best of our knowledge, this is the first report of $P$. shigelloides as a pathogen of $P$. pingi. This study will provide a rational framework for exploration of epidemiological analysis of $P$. shigelloides in fish diseases and would further benefit conservation of the species.
\end{abstract}

Keywords: Plesiomonas shigelloides, Percocypris pingi (Tchang, 1930), Identification, Genomic Analysis emerging enteric pathogen that is linked to intestinal and extra-intestinal infections in humans (González-Rey et al., 2011). It is recognized as a potential fish pathogen and has been isolated from red hybrid tilapia (Nadirah et al., 2012). In China, P. shigelloides is the pathogen of grass carp (Ctenopharyngodon idella), sturgeon (Huso huso $\times$ Acipenser ruthenus), gibel carp (Carassius auratus gibelio), black carp (Mylopharyngodon piceus) and tilapia (Oreochromis niloticus).

Percocypris pingi (Tchang, 1930), an endemic and commercially important species only found in the upper 
reaches of the Yangtze River in China, is a benthic fish that is typically found in rivers with torrential flow. The population of this fish decreased intensely due to overfishing, dam construction, habitat deterioration and water pollution, becoming endangered in the past decade. In May 2015, this species has been listed as Endangered (EN) in the Red List of China's Vertebrates (Li et al., 2016). Aquaculture may counteract this decline and improve biodiversity by sustaining healthy fish populations (Pan et al., 2016). Therefore, further research is essential to maintain sufficient production of $P$. pingi to meet fishing demands for conservation.

Recently, artificial propagation of $P$. pingi has been conducted in Sichuan and Yunnan provinces in China to restore their population and the hatchery-reared fish have been released into the river by several institutions (Li et al., 2016). Unfortunately, bacterial diseases are one of the major obstacles to the expansion of $P$. pingi farming. In 2016, an outbreak caused by $P$. shigelloides occurred in farmed $P$. pingi in China. However, the information about $P$. pingi disease is limited. In this study, we have described the isolation, identification and genomic analysis of $P$. shigelloides from diseased $P$. pingi. The aim of this study is to provide a theoretical foundation for further understanding of the epidemiology of $P$. shigelloides strains and ensure effective prevention and control measures.

\section{Materials and Methods}

\section{Sample Collection}

In August 2016, a disease outbreak in P. pingi (average body weight 45 gram) were obtained from a commercial source, which were cultured in a recirculating system in Lijiang city, Yunnan province, China. The culture water temperature was maintained at $24 \pm 1^{\circ} \mathrm{C}$. In the early stage of the disease, several fish began to show clinical signs including anorexia, caudal fin and skin ulceration and ascites (accumulation of abdominal fluid). Four days later, mortalities started to occur in this farm. Moribund fish were sampled and kidney tissues were incubated on Brain Heart Infusion (BHI, Difco Laboratories, Detroit, MI, USA) agar for bacterial isolation.

\section{Bacterial Isolation}

Tissue samples were aseptically collected from the kidney of moribund $P$. pingi $(\mathrm{N}=10)$ and incubated on BHI agar. Plates were incubated at $28^{\circ} \mathrm{C}$ for $24 \mathrm{~h}$. The bacterial colonies were re-streaked on $\mathrm{BHI}$ agar to obtain a pure culture. For long-term preservation the bacterial isolates were stored at $-80^{\circ} \mathrm{C}$ in sterile $\mathrm{BHI}$ containing $15 \%$ glycerol. In this study, a total of ten strains were isolated and two of them, were selected for further study.

\section{Molecular Characterization}

Genomic DNA from ten isolates was extracted using the EasyPure Genomic DNA Kit (Transgen Biotech, Beijing, China) in accordance with the manufacturer's instructions. A partial 16S rRNA gene sequence was amplified as described previously (Zhang et al., 2011). Briefly, universal PCR primers 27F (5'AGAGTTTGATCMTGGCTCAG-3') and 1492R (5'TACGGMTACCTTGTTACGACTT-3') were used for amplification of the 16S rRNA gene. In addition, primers PS23FW3 (5'-CTCCGAATACCGTAGAGTGCTATC C-3') and PS23RV3 (5'CTCCCCTACCCAATAACACCTAAA-3') were used for detection of the 23S rRNA gene of $P$. shigelloides (González-Rey et al., 2000).

\section{Biochemical Characteristics}

Of the ten isolates, two (strains LS1 and LL2) were identified based on biochemical characteristics, which were examined by API 20E system (bioMèrieux, Marcy l'Etoile, France) as reported previously (Krovacek et al., 2000) and by API strips (Chen et al., 2013). Hemolytic activity was assayed by spot inoculation of these strains on $5 \%$ sheep erythrocytes in BHI agar and erythrocyte lysis was recorded after $24 \mathrm{~h}$ of incubation at $37^{\circ} \mathrm{C}$.

\section{Pathogenicity Test}

In this study, zebrafish (Danio rerio) was used as the target fish to evaluate the pathogenicity of strain LS1 (Saralahti and Rämet, 2015). The isolate LS1 was used for the experimental infection of healthy zebrafish to assess their pathogenic potential. A total of 300 healthy zebrafish were randomly divided into seven groups (20 per group) and then allowed to acclimatize at $28^{\circ} \mathrm{C}$ for 7 $\mathrm{d}$ prior to challenge. Challenge assays were performed in triplicate. Isolate LS1 was cultured in BHI broth overnight, centrifuged and collected. The bacteria were resuspended in sterile PBS buffer at six different doses. Experimental groups were intraperitoneally inoculated with $20 \mu \mathrm{L}$ of cultured cells at doses of $3 \times 10^{8}, 3 \times 10^{7}$, $3 \times 10^{6}$ and $3 \times 10^{5} \mathrm{CFU} / \mathrm{mL}$, respectively. The zebrafish in the control group were injected with the same volume of PBS buffer. Clinical signs and mortality were recorded daily for $14 \mathrm{~d}$. In addition, bacteria were isolated from the moribund zebrafish and were detected by PCR assay based on the 23S rRNA gene of $P$. shigelloides. The statistical analysis was calculated using SPSS software (version 22.0) and the survival curve was performed by Origin software (version 9.0).

\section{Antimicrobial Susceptibility Testing}

In order to select the antimicrobial agents for treatment and define the antibiotic pattern of the isolates, antibiotic 
susceptibility of bacterial isolates was determined by the Kirby-Bauer disc-diffusion method. Escherichia coli ATCC25922 strain was used as control in the antimicrobial susceptibility tests. The sensitivity and resistance of the isolates and the zone diameter interpretive standards were determined in accordance with the CLSI (Clinical and Laboratory Standards Institute) criteria for animal isolates (CLSI, 2012). The susceptibility patterns of strains LS1 and LL2 against 24 antimicrobial agents (Oxoid, Mercers Row, Cambridge, UK), including acetylspiramycin, erythromycin, midecamycin, penicillin $\mathrm{G}$, ampicillin, cefazolin, ceftiofur, ceftriaxone, ceftazidime, ceftriaxone, tetracycline, doxycycline, enrofloxacin, nalidixic acid, enoxacin, tobramycin, streptomycin, spectinomycin, kanamycin, gentamicin, chloramphenicol, florfenicol, vancomycin and trimethoprim/sulfamethoxazole, were studied.

\section{Genome Sequencing, Assembly And Analysis}

The genome of the strain LS1 was sequenced using the Illumina HiSeq 4000 sequencing platform at Total Genomics Solution (TGS, Shenzhen, China). The SOAPdenovo (version 2.04) and GapCloser (version 1.12) software were used to assemble about $404 \mathrm{M}$ sequencing reads and obtain a genome of 3,866,061 bp that resulted in an estimated average 100 -fold coverage of the genome. The reads length was $150 \mathrm{bp}$ and the Q20 and Q30 clean data were $97.37 \%$ and $92.79 \%$, respectively. Reads assembly with the SOAPdenovo (version 2.04) and GapCloser (version 1.12) software yielded 51 scaffolds and 53 contigs. The genome was then screened using GeneMarks (version 4.6b) software, revealing 3,445 genes. The functions of encoded genes were annotated using the Virulence Factor of Pathogenic Bacteria Database (VFDB, www.mgc.ac.cn/VFs/main.htm) and the Antibiotic Resistance Genes Database (ARDB, http://ardb.cbcb.umd.edu/).

Phage detection was performed using the publicly available Phage Search Tool (PHAST) (http://phast.wishartlab.com/). Single nucleotide polymorphisms (SNPs) were detected using the MUMmer software (version 3.22) (Kurtz et al., 2004), the query sequence was aligned with the reference sequence (GenBank accession no. GCA_900087055.1). The variation sites between the query and reference sequences were identified and filtered preliminarily to detect potential SNP sites. The sequences with the length of $100 \mathrm{bp}$ at both sides of SNP in the reference sequence were extracted and aligned with assembly results to verify SNP sites using BLAT. If the length of the aligned sequence was shorter than $101 \mathrm{bp}$, the SNP was considered incredible and would be removed; if the extracted sequence could be aligned with the assembly results several times, the SNP was considered to be located in the repeat region and would be removed. BLAST, TRF (Version: 4.04) and Repeatmasker software (Version: 3.2.9) were used to predict SNPs in the repeat regions. The credible SNPs can be obtained by filtering those located in the repeat regions.

For comparative analysis, genome sequences of 3 reference $P$. shigelloides strains were selected, including those collected from water at an animal farm (GN7), from humans (302-73) and the NCTC 10360 strain as the type strain. The phylogenetic tree was constructed using the array of SNPs obtained from the strain LS1 and the reference strains. For each bacterium, all SNPs were connected with a similar order and the sequences with the same length were obtained as an input file in FASTA format. Then, the phylogenetic tree was constructed by TreeBeST (Nandi et al., 2010) using the method of PHYML and by setting bootstraps as 1,000 .

\section{Results}

\section{Isolation and Identification}

Ten isolates were isolated from ten diseased $P$. pingi. They were cultured, purified and identified as $P$. shigelloides by PCR detection based on 23S rRNA gene and 16S rRNA gene sequencing (González-Rey et al., 2000; Marathe et al., 2016). Sequence analysis revealed that the $16 \mathrm{~S}$ rRNA gene sequences for the ten isolates displayed $100 \%$ identity to the corresponding gene of $P$. shigelloides (NCTC 10360). The representative strains LS1 and LL2 were selected for further identification. Biochemical characteristics of the two strains were consistent with those of $P$. shigelloides (Table 1). The strains LS1 and LL2 did not show hemolysis on plates containing 5\% sheep blood agar and the lactose reaction result was negative for the two isolates (Table 1). The results revealed that strains LS1 and LL2 were identified as P. shigelloides.

\section{Pathogenicity to Zebrafish}

In the challenge trials, the isolate LS1 was pathogenic to zebrafish. Infected zebrafish began to die at $16 \mathrm{~h}$ postinoculation with a high dose and the most of the mortality occurred within $72 \mathrm{~h}$ (Fig. 1). The average cumulative mortalities of zebrafish were $90 \%, 50 \%, 30 \%$ and $18 \%$ at $3 \times 10^{8}, 3 \times 10^{7}, 3 \times 10^{6}$ and $3 \times 10^{5} \mathrm{CFU} / \mathrm{mL}$ within 7 days after challenge, respectively (Fig. 1). The moribund infected zebrafish showed clinical signs of hemorrhages at the base of the fins, fin ulceration, ascetic fluid and enlargement of the liver and gall bladder. Pure cultures of $P$. shigelloides were re-isolated from the kidneys of moribund fish. No clinical signs or bacteria were detected in control fish. Therefore, the isolate LS1 was a potential pathogen for fish. 
Table 1: Biochemical characteristics of strains LS1 and LL2 isolated from diseased P. pingi

\begin{tabular}{|c|c|c|c|}
\hline \multirow[b]{2}{*}{ Tests } & \multicolumn{2}{|c|}{ Reaction } & \multirow{2}{*}{$\begin{array}{l}\text { Reference } \\
P \text {. shigelloides* }\end{array}$} \\
\hline & LS1 & LL2 & \\
\hline$\beta$-galactosidase & + & + & + \\
\hline Arginine dihydrolase & + & + & + \\
\hline Lysine decarboxylase & + & + & + \\
\hline Ornithine decarboxylase & + & + & + \\
\hline Citrate utilization & - & - & - \\
\hline $\mathrm{H}_{2} \mathrm{~S}$ production & - & - & - \\
\hline Urease & - & - & - \\
\hline Tryptophan deaminase & - & - & - \\
\hline Indole production & + & + & + \\
\hline V-P reaction & - & - & - \\
\hline Gelatin & - & - & - \\
\hline Glucose & + & + & + \\
\hline Mannitol & - & - & - \\
\hline Inositol & + & + & + \\
\hline Sorbitol & - & - & - \\
\hline Rhamnose & - & - & - \\
\hline Sucrose & - & - & - \\
\hline Melibiose & - & - & - \\
\hline Amygdalin & - & - & - \\
\hline L-arabinose & - & - & - \\
\hline Oxidase & + & + & + \\
\hline Lactose & - & - & - \\
\hline
\end{tabular}

Notes: + , positive; - , negative

*Desicription in the Bergey's manual of systematic bacteriology $\left(9^{\text {th }}\right)$

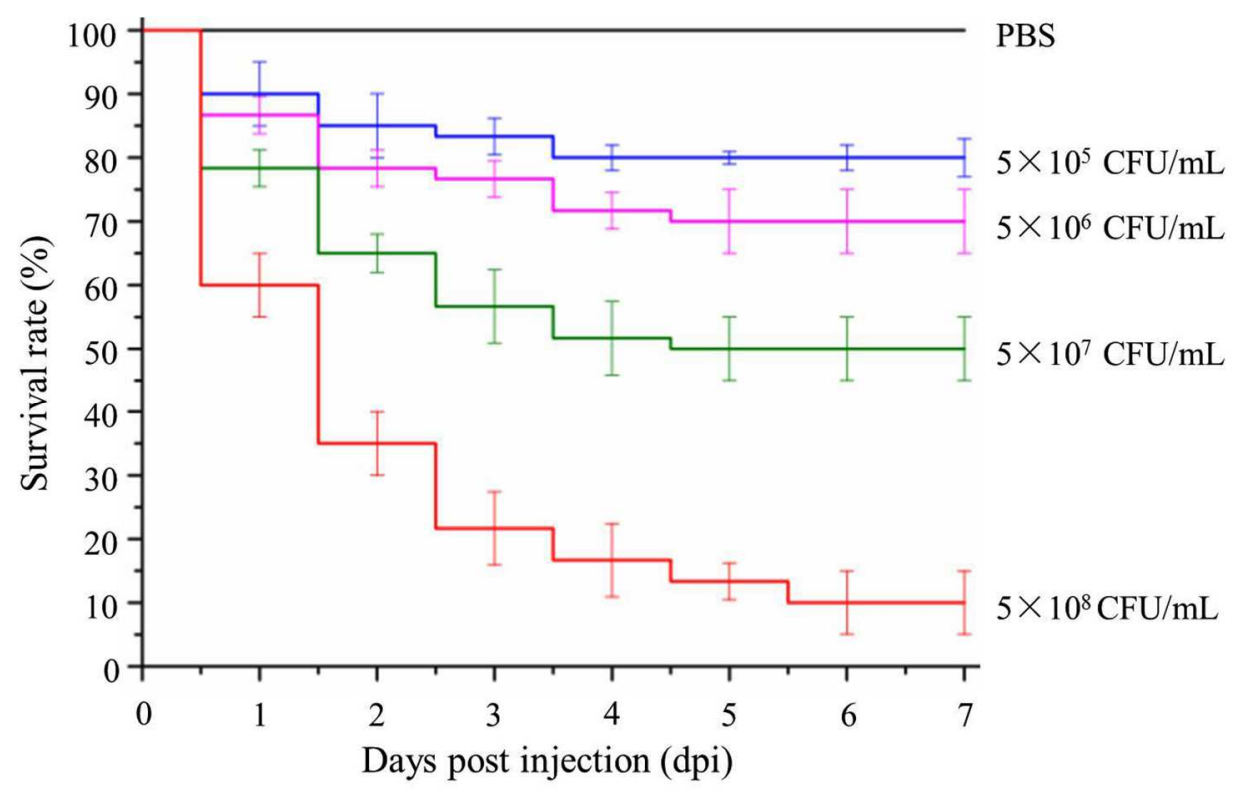

Fig. 1: Survival curve of zebrafish infected with $P$. shigelloides

\section{Antimicrobial Susceptibility}

Antibiotic susceptibility tests indicated that LS1 and LL2 isolates were resistant to ampicillin, penicillin G, trimethoprim/sulfamethoxazole, tetracycline, enrofloxacin, nalidixic acid and enoxacin; mid-resistant to acetylspiramycin; and susceptible to other antibiotics (Table 2). These results indicated that strains LS1 and LL2 were multi-drug resistant strains. 


\section{Genome Features and Genomic Analysis}

Genome sequence was assemble and contained a total of 3,866,061 bp. The whole genome contains 3,445 genes that account for $83.27 \%$ of the genome. The ARDB annotated 3 genes conferring resistance to tetracycline (tetA), trimethoprim (dfra) and bacitracin (baca). Strain LS1 contained 10 prophage sequences with a total length of $190,791 \mathrm{bp}$, including 2 intact prophages and 8 incomplete prophages. The draft genome sequences for $P$. shigelloides LS1 have been deposited at GenBank under accession no. MUNJ00000000.
Compared to the reference strain NCTC 10360, strains LS1, 302-73 and GN7 contained 65,685, 65,997 and 66,859 SNPs, respectively. For the strain LS1, approximately $87.8 \%$ of the SNPs within coding sequences resulted in synonymous changes at the protein level and $18.0 \%$ of the total SNPs were intergenic mutants. In the phylogenetic tree, strains LS1 and GN7 were grouped into one evolutionary branch (Fig. 2). The results revealed that strain LS1 was closely related to strain GN7 at the genome level, which was isolated from water at an animal farm.

Table 2: Susceptibility of strains LS1 and LL2 to 24 antimicrobial agents

\begin{tabular}{|c|c|c|c|}
\hline \multirow[b]{2}{*}{ Antimicrobial agents } & \multirow[b]{2}{*}{ Content $(\mu \mathrm{g})$} & \multicolumn{2}{|c|}{ Susceptibility } \\
\hline & & LS1 & LL2 \\
\hline Acetylspiramycin & 30 & I & I \\
\hline Erythromycin & 15 & $\mathrm{~S}$ & $\mathrm{~S}$ \\
\hline Midecamycin & 30 & $\mathrm{~S}$ & $\mathrm{~S}$ \\
\hline Penicillin G & $10 \mathrm{U}$ & $\mathrm{R}$ & $\mathrm{R}$ \\
\hline Ampicillin & 10 & $\mathrm{R}$ & $\mathrm{R}$ \\
\hline Cefazolin & 30 & $\mathrm{~S}$ & $\mathrm{~S}$ \\
\hline Ceftiofur & 30 & $\mathrm{~S}$ & $\mathrm{~S}$ \\
\hline Ceftriaxone & 30 & $\mathrm{~S}$ & $\mathrm{~S}$ \\
\hline Ceftazidime & 30 & $\mathrm{~S}$ & $\mathrm{~S}$ \\
\hline Cefoperazone & 75 & $\mathrm{~S}$ & $\mathrm{~S}$ \\
\hline Tetracycline & 30 & $\mathrm{R}$ & $\mathrm{R}$ \\
\hline Doxycycline & 30 & $\mathrm{~S}$ & $\mathrm{~S}$ \\
\hline Enrofloxacin & 5 & $\mathrm{R}$ & $\mathrm{R}$ \\
\hline Nalidixic acid & 30 & $\mathrm{R}$ & $\mathrm{R}$ \\
\hline Enoxacin & 10 & $\mathrm{R}$ & $\mathrm{R}$ \\
\hline Tobramycin & 10 & $\mathrm{~S}$ & $\mathrm{~S}$ \\
\hline Streptomycin & 10 & $\mathrm{~S}$ & $\mathrm{~S}$ \\
\hline Spectinomycin & 100 & $\mathrm{~S}$ & $\mathrm{~S}$ \\
\hline Kanamycin & 30 & $\mathrm{~S}$ & $\mathrm{~S}$ \\
\hline Gentamicin & 10 & $\mathrm{~S}$ & $\mathrm{~S}$ \\
\hline Chloramphenicol & 30 & $\mathrm{~S}$ & $\mathrm{~S}$ \\
\hline Florfenicol & 30 & $\mathrm{~S}$ & $\mathrm{~S}$ \\
\hline Vancomycin & 30 & $\mathrm{~S}$ & $\mathrm{~S}$ \\
\hline Trimethoprim/Sulfamethoxazole & 300 & $\mathrm{R}$ & $\mathrm{R}$ \\
\hline
\end{tabular}

Notes: S, sensitivity; I, intermediate resistance; R, resistance. U, unit

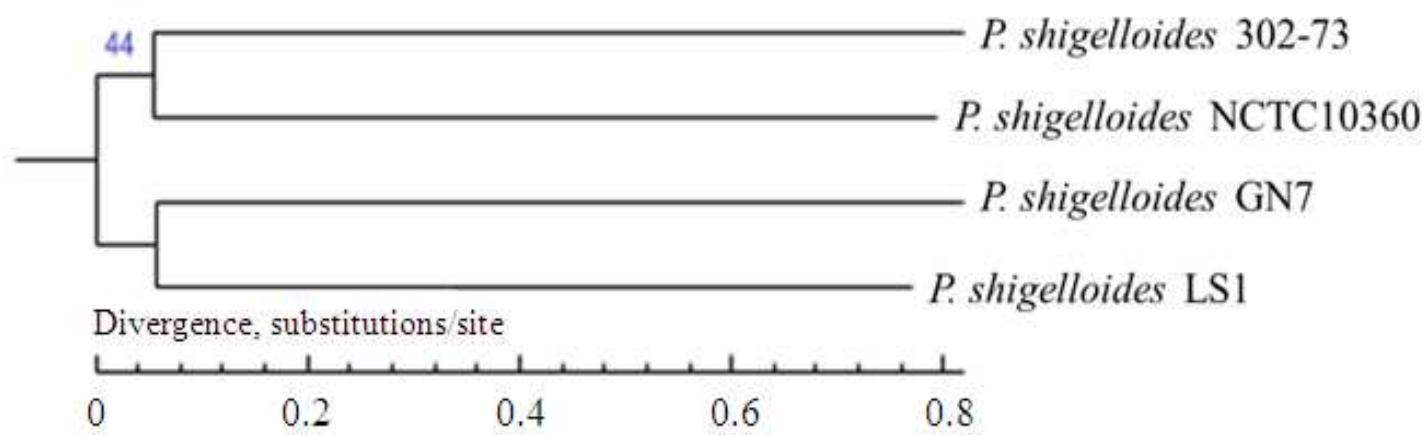

Fig. 2: The phylogenetic tree of $P$. shigelloides based on SNPs 


\section{Discussion}

In this study, ten bacterial strains with identical morphology were isolated from diseased $P$. pingi cultured in Yunnan province, China. LS1 and LL2, two of the ten isolates, were identified as P. shigelloides, according to general biochemical characteristics and $16 \mathrm{~S}$ rRNA gene sequencing. The $16 \mathrm{~S}$ rRNA sequencing analysis also indicated a very close relationship with $P$. shigelloides $(100 \%$, for GenBank accession No. LT575468). Furthermore, species-specific PCR confirmed that the isolates were $P$. shigelloides (González-Rey et al., 2000). The strain LS1 caused a high mortality at dose $\geq 3 \times 10^{7} \mathrm{CFU} / \mathrm{mL}$, indicating that isolate LS1 was strongly virulent to zebrafish. The results suggested that $P$. shigelloides was an important pathogen of $P$. pingi. For the purpose of conserving the population of $P$. pingi, more studies should be carried out on diseases caused by $P$. shigelloides.

Plesiomonas shigelloides, an emerging pathogen of humans and animals, is widespread in the aquatic environment. They have been isolated from various different animals, such as shellfish, freshwater fish, snakes, dogs, goats, cats, swine and monkeys (González-Rey et al., 2011). Interestingly, not all farmed fishes were infected with $P$. shigelloides, however, this bacterium is a common source of disease in grass carp, tilapia, Chinese giant salamander (Andrias davidianus) and black carp in China. To the best of our knowledge, this is the first report on P. shigelloides as a pathogen of $P$. pingi. The present results showed that $P$. shigelloides as an important pathogen to $P$. pingi. And the $P$. pingi is susceptible to $P$. shigelloides infection, resulting in high mortality. In the present study, two weeks after the initial onset of $P$. shigelloides infection, mortality levels had reached $50 \%$.

The results of antibacterial drug sensitivity tests suggested that the two selected isolates of $P$. shigelloides were sensitive to most of the antibiotics, but resistant to ampicillin, tetracycline and trimethoprim/sulfamethoxazole. Furthermore, isolate LS1 contained the tetracycline resistance gene tetA and the trimethoprim/sulfamethoxazole resistance gene $d f r a$ according to ARDB annotated. Importantly, these results were similar to those for isolates from Asian arowana (Scleropages formosus) in Korea (Jun et al., 2011). P. shigelloides is mostly resistant to penicillin and ampicillin, whereas it is usually sensitive to the third-generation cephalosporin as reported previously (Brenden et al., 1988). Similar to the report by Brenden et al. (1988), strains LS1 and LL2 were resistant to penicillin and ampicillin, but were sensitive to ceftriaxone, ceftazidime and cefoperazone.

In this study, control of $P$. shigelloides infection in $P$. pingi was based on treatment with doxycycline, so that after one week, the mortality of $P$. pingi was effectively controlled and was less than $5 \%$. In addition, water is the major source of contamination with $P$. shigelloides and an important vehicle for the spread of this species (Kim et al., 2015). Therefore, it is important to control the abundance of $P$. shigelloides in water for prevent $P$. shigelloides infection in $P$. pingi.

In the present study, we confirmed the isolation, identification and genomic analysis of $P$. shigelloides isolated from diseased $P$. pingi reared to restore their population in China. We found that $P$. shigelloides was pathogenic to zebrafish and it possessed 3 antibioticresistant genes in the genome sequence. Furthermore, to investigate the phylogenetic relationships among these four strains, we used the neighbor-joining method to construct a phylogenetic tree using the SNPs. Strains GN7, LS1 and NCTC10360 belong to the same evolutionary clade and strain LS1 was found to be closely related to strain GN7, which was isolated from animal farm. The results indicated that $P$. shigelloides strains from an animal could be transmitted to fish. Given that $P$. ping $i$ is a near endangered cyprinid species, the results would be provide some effective prevention and control strategies for the farm of $P$. pingi and would further benefit conservation of the species.

\section{Acknowledgement}

The authors thank Ph.D. Xujie Zhang for his adivce relating to the analysis of biochemical characteristics.

\section{Funding Information}

This study was supported by the Project (2017FB01) of State Key Laboratory of Freshwater Ecology and Biotechnology, the Project (2016NSG01) of State key laboratory of water resources and hydropower engineering science (Wuhan University); the National Natural Science Foundation of China (Grant No. 51439006) and the Project (2016A001) of Hubei Key Laboratory of Regional Development and Environmental Response (Hubei University).

\section{Author's Contributions}

Lei Pan and Shuiyi Liu: Contributed to the planning and implementation of this study as well as interpretation of article preparation and drafted the manuscript.

Xuwei Cheng and Yiting Tao: Coordinated the dataanalysis and contributed to the writing of the manuscript.

Tao Yang, Peipei Li, Zhengxiang Wang and Dongguo Shao: Designed the research plan and participated in the experiments.

Defeng Zhang: Contributed to the planning and implementation of research work, and revising the article. 


\section{Ethics}

Animal experiments were carried out according to animal welfare standards and were approved by the Ethical Committee for Animal Experiments of the Pearl River Fisheries Research Institute, Chinese Academy of Fishery Sciences. All animal experiments were in compliance with the guidelines of the Animal Welfare Council of China.

\section{Conflict of Interest}

The authors declare that they have no competing interests. The corresponding author affirms that all of the authors have read and approved the manuscript.

\section{References}

Alexander, S., M. Fazal, E. Burnett, A. Deheer-Graham and K. Oliver et al., 2016. Complete genome sequence of Plesiomonas shigelloides type strain NCTC10360. Genome Announc., 4: e01031-16. DOI: $10.1128 /$ genomeA.01031-16

Brenden, R.A., M.A. Miller and J.M. Janda, 1988. Clinical disease spectrum and pathogenic factors associated with Plesiomonas shigelloides infections in humans. Rev. Infectious Dis., 10: 303-316.

Brenner, D J., N.R. Krieg, Staley and J.T. Plesiomonas, 2005. Bergey's Manual of Systematic Bacteriology. In: The Proteobacteria: The Gammaproteobacteria, Brenner, D.J. (Ed.), Springer, New York, pp: 740-744.

Chen, X., Y. Chen, Q. Yang, H. Kong and F. Yu et al., 2013. Plesiomonas shigelloides infection in Southeast China. PLoS One, 8: e77877- e77877. DOI: 10.1371/journal.pone.0077877

CLSI, 2012. Methods for Antimicrobial Dilution and Disk Susceptibility Testing of Infrequently Isolated or Fastidious Bacteria: Approved Guideline. 1st Edn., Clinical and Laboratory Standards Institute, Wayne, ISBN-10: 1562386077, pp: 61.

González-Rey, C., S.B. Svenson, L. Bravo, J. Rosinsky and I. Ciznar et al., 2000. Specific detection of Plesiomonas shigelloides isolated from aquatic environments, animals and human diarrhoeal cases by PCR based on 23S rRNA gene. FEMS Immunol. Med. Microbiol., 29: 107-113.

González-Rey, C., A. Siitonen, A. Pavlova, I. Ciznar and S.B. Svenson et al., 2011. Molecular evidence of Plesiomonas shigelloides as a possible zoonotic agent. Folia Microbiol., 56: 178-184. DOI: $10.1007 / \mathrm{s} 12223-011-0032-2$

Jun, J.W., J.H. Kim, C.H. Choresca and S. Chang, 2011. Isolation and molecular detection of Plesiomonas shigelloides containing tetA gene from Asian arowana (Scleropages formosus) in a Korean aquarium. African J. Microbiol. Res., 5: 5019-5021.
Kim, K., S. Lee and D. Kwak, 2015. Prevalence, biochemical characteristics and antibiotic susceptibility of Aeromonas, Vibrio and Plesiomonas isolated from different sources at a zoo. J. Zoo Wildlife Med., 46: 298-305.

Krovacek, K., L.M. Eriksson, C. González-Rey, J. Rosinsky and I. Ciznarc et al., 2000. Isolation, biochemical and serological characterisation of Plesiomonas shigelloides from freshwater in Northern Europe. Comparative Immunol., Microbiol. Infectious Dis., 23: 45-51.

Kurtz, S., A. Phillippy, A.L. Delcher, M. Smoot and M. Shumway et al., 2004. Versatile and open software for comparing large genomes. Genome Biol. DOI: $10.1186 / \mathrm{gb}-2004-5-2-\mathrm{r} 12$

Li, X., Y. Deng, K. Yang, W. Gan and R. Zeng et al., 2016. Genetic diversity and structure analysis of Percocypris pingi (Cypriniformes: Cyprinidae): implications for conservation and hatchery release in the Yalong River. PLoS ONE. DOI: 10.1371/journal.pone.0166769

Marathe, N.P., S.S. Gaikwad, A.A. Vaishampayan, M.H. Rasane and Y.S. Shouche, 2016. Mossambicus tilapia (Oreochromis mossambicus) collected from water bodies impacted by urban waste carries extended-spectrum beta-lactamases and integronbearing gut bacteria. J. Biosci., 41: 341-346. DOI: $10.1007 / \mathrm{s} 12038-016-9620-2$

Nadirah, M., H.H. Ruhil, K.C. Jalal and M. Najiah, 2012. Occurrence of Plesiomonas shigelloides in cultured red hybrid tilapia (Oreochromis niloticus) from tropical rivers, east coast Malaysia. Pak. J. Biol. Sci., 15: 600-603.

Nandi, T., C. Ong, A.P. Singh, J. Boddey and T. Atkins et al., 2010. A genomic survey of positive selection in Burkholderia pseudomallei provides insights into the evolution of accidental virulence. PLoS Pathogens. DOI: 10.1371/journal.ppat.1000845

Pan, L., J. Hu, C. Peng, H. Liu and Z. Zhang et al., 2016. Use of magnetic resonance imaging to assess ovarian maturation in live Rhinogobio ventralis (Sauvage \& Dabry de Thiersant, 1874). Theriogenology, 86: 1969-1974. DOI: $10.1016 /$ j.theriogenology.2016.06.015

Salerno, A., I. Čižnár, K. Krovacek, M. Conte and S. Dumontet et al., 2010. Phenotypic characterization and putative virulence factors of human, animal and environmental isolates of Plesiomonas shigelloides. Folia Microbiol., 55: 641-647. DOI: 10.1007/s12223-010-0104-8

Saralahti, A. and M. Rämet, 2015. Zebrafish and streptococcal infections. Scandinavian J. Immunol., 82: 174-183. DOI: $10.1111 /$ sji.12320

Zhang, X.J., G.M. Qin, X.W. Bing, L. Yan and L.G. Liang, 2011. Molecular and phenotypic characterization of Vibrio aestuarianus, a pathogen of the cultured tongue sole, Cynoglossus semilaevis Gunther. J. Fish Dis., 34: 57-64. DOI: 10.1111/j.1365-2761.2010.01212.x 